\title{
Characterization of Nanoimprint Resin and Antisticking Layer by Scanning Probe Microscopy
}

\author{
Makoto Okada ${ }^{1,3}$, Yuji Kang1,3 ${ }^{1,}$ Ken-ichiro Nakamatsu, 1, 4, Masayuki Iwasa², \\ Kazuhiro Kanda ${ }^{1,3}$, Yuichi Haruyama ${ }^{1,3}$, and Shinji Matsui ${ }^{1,3}$ \\ ${ }^{1}$ Graduate School of Science, LASTI, University of Hyogo \\ 3-1-2 Koto, Kamigori, Ako, Hyogo 678-1205, Japan \\ ${ }^{2}$ SII Nanotechnology Inc., RBM, Tsukiji Bldg. 2-15-5 Shintomi, Chuo-ku, Tokyo 104-0041, Japan \\ ${ }^{3}$ JST -CREST, Sanbancho, Chiyoda-ku, Tokyo, 102-0075, Japan \\ ${ }^{4}$ JSPS, 6 Ichibancho, Chiyoda-ku, Tokyo 102-8471, Japan \\ E-mail:m.okada@lasti.u-hyogo.ac.jp
}

Keywords: nanoimprint lithography, scanning probe microscopy, antisticking layer, nanoimprint resin

\section{Introduction}

In nanoimprint lithography (NIL) ${ }^{1-3)}$, a fluorinated self-assembled monolayer (F-SAM) is mainly used as an antisticking layer. The sidewalls of a nanostructure mold cause friction with NIL resist during demolding, as shown in Figure1. In addition, adhesion occurs between the upper and bottom surfaces of the nanostructure mold and resist. We assume that the frictional and adhesion forces are main factor in demolding force.

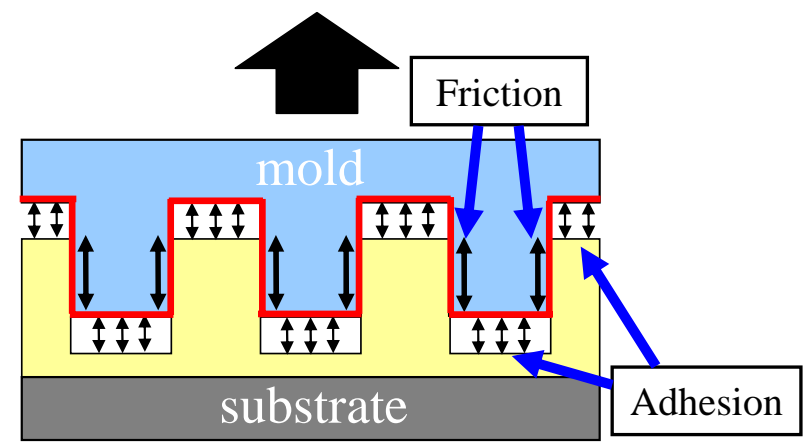

Fig. 1 Schematic of demolding process

We measured the frictional and adhesion forces by scanning probe microscopy (SPM) using Si cantilevers with and without the F-SAM ${ }^{46)}$ to evaluate the releasing effect of F-SAM for NIL resists. Furthermore, we evaluated the releasing effect of a release-agent spray-coated hydrogen silsesquioxane (HSQ) to carry out room-temperature nanoimprint (RT-NIL) ${ }^{7)}$ using a mold without the F-SAM onto it as a resist.

\section{Experiments}

We used the SPM system (E-sweep/NanoNavi Station: SII NanoTechnology Inc.) which can be used to control the experimentation environment such as the vacuum, temperature, and air humidity in this experiment. The adsorbed water on the sample affects the measurement of frictional and adhesion forces by SPM. We therefore carried out SPM measurements with the air humidity at around $80 \%$ to uniform the effect of the adsorbed water. We used a silane-coupling agent with fluoropolymer (OPTOOL DSX: Daikin industries) as the antisticking layer. ${ }^{8)}$ The structural formula of the silane-coupling agent is $\mathrm{C}_{3} \mathrm{~F}_{7}-\left(\mathrm{OCF}_{2} \mathrm{CF}_{2} \mathrm{CF}_{2}\right)_{\mathrm{n}} \mathrm{OC}_{2} \mathrm{~F}_{4} \mathrm{C}_{2} \mathrm{H}_{4}-\mathrm{Si}\left(\mathrm{OCH}_{3}\right)_{3}$. PMMA (OEBR 1000: TOKYO OHKA KOGYO Co.) and PAK-01 (Toyo Gosei Co.) were used as the thermoplastic and UV curable resists, respectively. The contact force was about $10 \mathrm{nN}$.

\section{Result and Discussins}

Figure 2(a) shows the frictional forces of PMMA and PAK-01. 

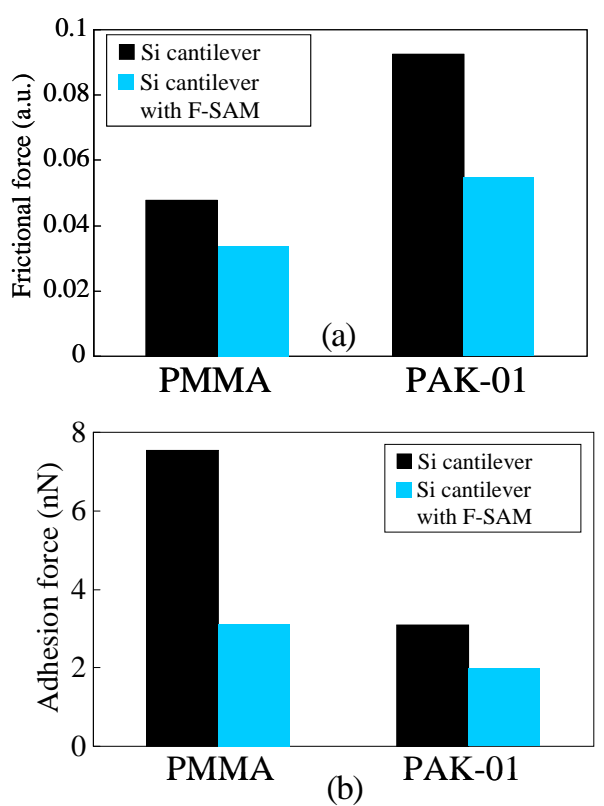

Fig. 2 (a) Frictional and (b) adhesion forces of PMMA and PAK-01 measured by SPM using Si cantilevers with and without F-SAM.

The frictional force measured using the cantilever with the F-SAM of PMMA and PAK-01 are respectively 0.7 and 0.6 times lower than those using the non-coated cantilever. Figure 2(b) shows the adhesion forces of PMMA and PKA-01. The adhesion forces of PMMA and PAK-01 measured by SPM using the cantilever with the F-SAM are respectively 0.4 and 0.6 times lower than those using the cantilever. These results show that SPM measurement using the cantilever with and without the F-SAM can be applied to evaluate the nanoscale release effect between an antisticking layer and NIL resists.

It is known that the F-SAM deteriorates due to repeated nanoimprinting. ${ }^{9)}$ To prevent the F-SAM coated on the NIL mold from deteriorating, we proposed the new RT-NIL using a release-agent spray-coated HSQ. ${ }^{7)}$ The schematic of conventional and proposed RT-NIL process are shown in Fig. 3.

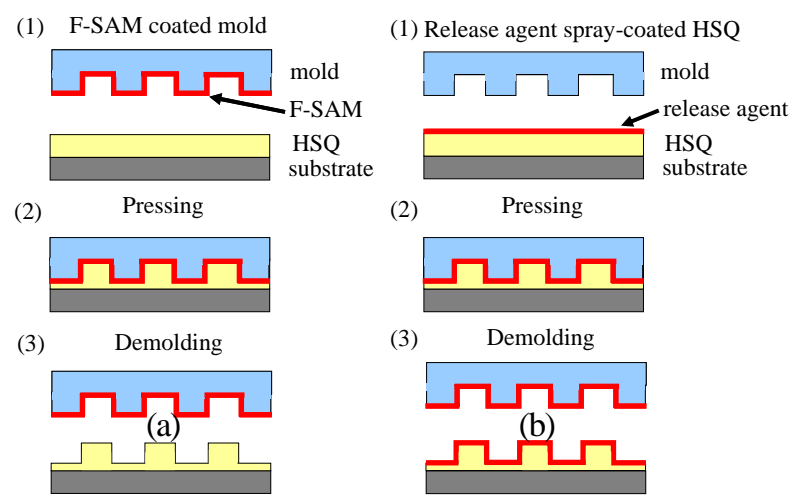

Fig. 3 Schematics of (a) conventional and (b) proposed methods in RT-NIL.
In the proposed process, we carried out RT-NIL on the release-agent spray-coated HSQ using a mold without the F-SAM. We first measured frictional and adhesion forces of HSQ and the release-agent spray-coated HSQ by SPM using the Si cantilevers with and without the F-SAM to evaluate the releasing effect of the release-agent spray-coated HSQ. The measurement results of frictional and adhesion forces in those cases are shown in Figs. 4(a) and 4(b), respectively. The contact force was about 0.6 $\mathrm{nN}$.

Figures 4(1) and 4(2) show frictional and adhesion forces between HSQ and Si cantilever without and with the F-SAM. Figure 4(3) indicates these forces between the release-agent spray-coated HSQ and Si cantilever. Both frictional and adhesion forces of (2), (3) in Fig 4 were lower than those of Fig. 4(1). In addition, the frictional and adhesion forces of using the Si cantilever on the release-agent spry-coated HSQ surface was nearly the same as those of using the F-SAM coated cantilever on HSQ surface.

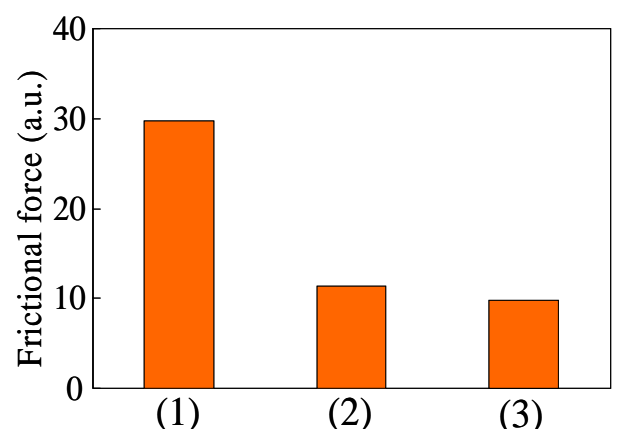

(a)

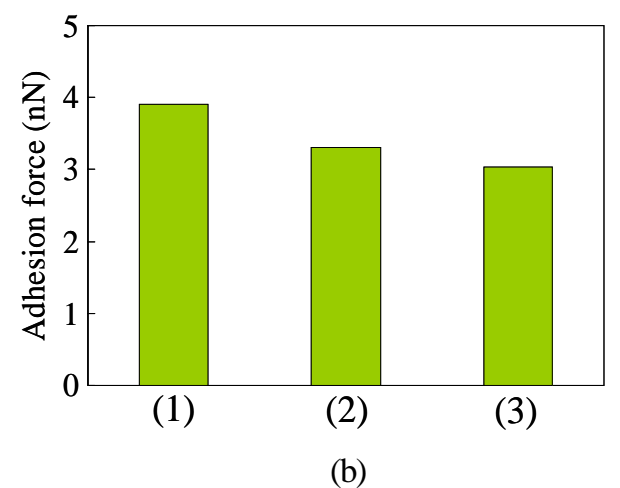

Fig. 4 (a) Frictional and (b) adhesion forces of HSQ and release-agent spray-caoted HSQ measured by SPM using $\mathrm{Si}$ cantilevers with and without F-SAM.

We therefore tried to RT-NIL on the release-agent spray-coated HSQ using the mold without the F-SAM. The imprinting pressure and time were 40MPa and $2 \mathrm{~min}$. Figure 5(a) shows scanning electron microscopy (SEM) 
image of the mold (180-nm line and 220-nm space) and 5(b) shows image of the HSQ imprinted pattern. This nanoimprinting was successfully carried out without the resist adhering to the mold.

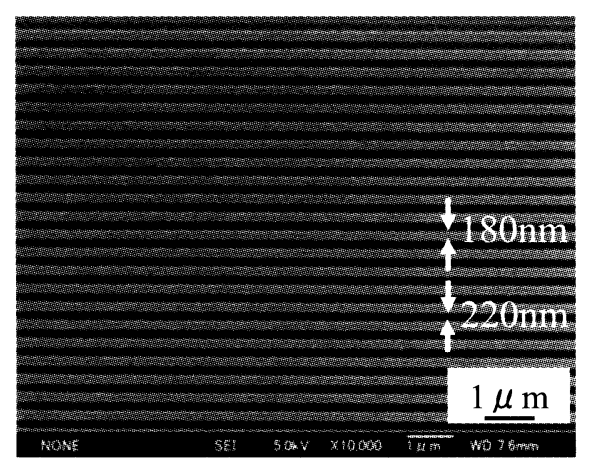

(a)

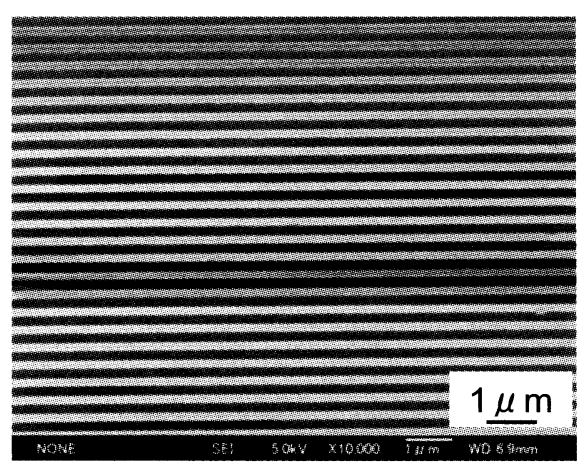

(b)

Fig. 5 SEM images of (a) mold and (b) HSQ imprinted patterns.

\section{Conclusion}

We carried out SPM measurements using the $\mathrm{Si}$ cantilevers with and without the F-SAM on the NIL resists. The measurement results using the cantilever with the F-SAM showed a lower frictional and adhesion forces than the results for which only the cantilever was used.
We next evaluate the releasing effect of the release-agent spray-coated HSQ by using SPM measurements.

Furthermore, we confirmed from the measurement results that the frictional and adhesion forces between the Si cantilever and the release-agent spray-coated HSQ were nearly the same value as those between the F-SAM cantilever and HSQ. In fact, nanoimprinting on the release-agent spray-coated HSQ using the mold without the F-SAM was successfully carried out without the resist adhering to the mold.

In this way, we have demonstrated that SPM measurement using Si cantilevers with and without the F-SAM is very useful to evaluate nanoscale releasing effect between F-SAM and resist in NIL.

\section{References}

1) S. Y. Chou, P. R. Krauss, and P. J. Renstrom: Appl. Phys. Lett., 67 (1995) 3114

2) S. Y. Chou, P. R. Krauss, and P. J. Renstrom: Science, 272 (1996) 85

3) S. Y. Chou, P. R. Krauss, W. Zhang, L. Guo, and L. Zhuang: J. Vac. Sci. Technol. B, 15 (1997) 2897

4) C. M. Mate, G M. McClelland, R. Erlandsson, and S. Chiang: Phys. Rev. Lett., 59 (1987) 1942

5) H. A. Mizes, K.-G Loh, R. J. D. Miller, S. K. Ahuja, and E. F. Grabowski: Appl. Phys. Lett., 59 (1991) 2901

6) J. Tallal, M. Gordon, K. Berton. A. L. Charley and D. Peyrade : Microelectronic Engineering, 83 (2006) 851

7) M. Okada, K. Nakamatsu, M. Iwasa, K. Kanda, Y. Haruyama, and S. Matsui: Appl. Phys. Exp., 2 (2009) $016502 \mathrm{~N}$.

8) Y. Hirai, S. Yoshida, A. Okamoto, Y. Tanaka, M. Endo, S. Irie, H. Nakagawa, and M. Sasago: J. Photopolym. Sci. Technol., 14 (2001) 457

9) Y. Tada, H. Yoshida, and A. Miyauchi: J. Photopolym. Sci. Technol., 20 (2007) 545 